\title{
Certain generating functions for the quadruple hypergeometric series $K_{10}$
}

\author{
Praveen Agarwal ${ }^{1,2}$, Jihad A. Younis ${ }^{3}$ and Taekyun Kim ${ }^{4}$ \\ ${ }^{1}$ Department of Mathematics, Anand International College of Engineering \\ Jaipur-303012, India \\ ${ }^{2}$ Department of Mathematics, Harish-Chandra Research Institute (HRI) \\ Allahbad-211 019, UP, India \\ e-mail: goyal.praveen2011@gmail.com \\ ${ }^{3}$ Department of Mathematics, Aden University \\ Aden, Yemen \\ e-mail: jihadalsaqqaf@gmail.com \\ ${ }^{4}$ Department of Mathematics, College of Natural Science \\ Kwangwoon University, Seoul 139-704, South Korea \\ e-mail: tkkimekw.ac.kr
}

Received: 2 September 2019 Accepted: 14 November 2019

\begin{abstract}
In this work we aim at establishing certain generating relations, involving the Exton function of four variables $K_{10}$. Some special cases of the main results here are also considered.
\end{abstract} Keywords: Laplace integral, Quadruple hypergeometric series, Generating functions. 2010 Mathematics Subject Classification: 33C50, 33C56.

\section{Introduction}

The Gaussian hypergeometric function is defined by

$$
{ }_{2} F_{1}(a, b ; c ; z)=\sum_{n=0}^{\infty} \frac{(a)_{n}(b)_{n}}{(c)_{n}} \frac{z^{n}}{n !}, \quad(|x|<1),
$$

(see $[9,10])$, where $(a)_{n}$ denotes the Pochhammer symbol given by 


$$
(a)_{n}=\frac{\Gamma(a+n)}{\Gamma(a)}=a(a+1) \cdots(a+n-1), \quad(n \in \mathbb{N}:=\{1,2,3, \cdots\})
$$

and

$$
(a)_{0}=1 .
$$

An interesting further generalization of the series ${ }_{2} F_{1}$ is due to Exton [5] who defined the following hypergeometric series in four variables:

$$
\begin{aligned}
K_{10}\left(a_{1}, a_{1}, a_{1}, a_{1}, a_{2}, a_{2}, a_{3}, a_{4} ; c_{1}, c_{2}, c_{3}, c_{4}, ; x, y, z, u\right) \\
=\sum_{m, n, p, q=0}^{\infty} \frac{\left(a_{1}\right)_{m+n+p+q}\left(a_{2}\right)_{m+n}\left(a_{3}\right)_{p}\left(a_{4}\right)_{q}}{\left(c_{1}\right)_{m}\left(c_{2}\right)_{n}\left(c_{3}\right)_{p}\left(c_{4}\right)_{q}} \frac{x^{m}}{m !} \frac{y^{n}}{n !} \frac{z^{p}}{p !} \frac{u^{q}}{q !} .
\end{aligned}
$$

By suitable adjustment of parameters and variables in $K_{10}$, we can easily find that $K_{10}$ is unification and generalization of Lauricella's functions $F_{A}^{(3)}$ and $F_{E}[6,9]$ and Appell's functions $F_{2}$ and $F_{4}[9,10]$. The following is the Laplace integral representation of the function $K_{10}$ (see [5]):

$$
\begin{aligned}
& K_{10}\left(a_{1}, a_{1}, a_{1}, a_{1}, a_{2}, a_{2}, a_{3}, a_{4} ; c_{1}, c_{2}, c_{3}, c_{4} ; x, y, z, u\right)=\frac{1}{\Gamma\left(a_{1}\right)} \int_{0}^{\infty} e^{-s} \\
& \times s^{a_{1}-1} \Psi_{2}\left(a_{2} ; c_{1}, c_{2} ; s x, s y\right)_{1} F_{1}\left(a_{3} ; c_{3} ; s z\right){ }_{1} F_{1}\left(a_{4} ; c_{4} ; s u\right) d s,\left(\operatorname{Re}\left(a_{1}>0\right),\right.
\end{aligned}
$$

where ${ }_{1} F_{1}$ is Kummer's function and $\Psi_{2}$ is Humbert function defined, respectively, by

$$
{ }_{1} F_{1}(a ; b ; x)=\sum_{n=0}^{\infty} \frac{(a)_{n}}{(b)_{n}} \frac{x^{n}}{n !}, \quad|x|<\infty
$$

and

$$
\Psi_{2}(a, b, c ; x, y)=\sum_{m, n=0}^{\infty} \frac{(a)_{m+n}}{(b)_{m}(c)_{n}} \frac{x^{m}}{m !} \frac{y^{n}}{n !}, \quad|x|<\infty,|y|<\infty .
$$

In various areas of applied mathematics and mathematical physics, generating functions play an important role in the investigation of various useful properties of the sequences which they generate. Generating functions are used to find certain properties and formulas for numbers and polynomials in a wide range of research subjects such as modern combinatorics (see, e.g., [10]). In fact, a remarkably large number of generating functions involving a variety of hypergeometric functions have been developed by many authors (for example, [1, 2, 3, 8]), here, we aim at establishing ten new generating functions for the Exton hypergeometric function $K_{10}$. Some special cases of the main results here are also considered. 


\section{Generating functions}

Here certain generating relations for the Exton hypergeometric function $K_{10}$ are presented.

$$
\begin{aligned}
\sum_{k=0}^{\infty} \frac{w^{k}}{k !} K_{10}\left(a_{1}+k, a_{1}+k, a_{1}+k, a_{1}+k, a_{2}, a_{2}, a_{3}, a_{4} ; c_{1}, c_{2}, c_{3}, c_{4} ; x, y, z, u\right) \\
=(1-z-u)^{-a_{1}} \sum_{k=0}^{\infty} \frac{1}{k !}\left(\frac{w}{(1-z-u)}\right)^{k} K_{10}\left(a_{1}+k, a_{1}+k, a_{1}+k, a_{1}+k, a_{2}, a_{2},\right. \\
\left.\quad c_{3}-a_{3}, c_{4}-a_{4} ; c_{1}, c_{2}, c_{3}, c_{4} ; \lambda_{1} x, \lambda_{1} y, \lambda_{2} z, \lambda_{2} u\right),
\end{aligned}
$$

where $\lambda_{1}=\frac{1}{1-z-u}, \lambda_{2}=\frac{1}{z+u-1}$.

$$
\begin{aligned}
\sum_{k=0}^{\infty} & \frac{w^{k}}{k !} K_{10}\left(a_{1}+k, a_{1}+k, a_{1}+k, a_{1}+k, a_{2}, a_{2}, a_{3}, a_{4} ; c_{1}, c_{2}, c_{3}, c_{4} ; x, y, z, u\right) \\
= & (1-u)^{-a_{1}} \sum_{k=0}^{\infty} \frac{1}{k !}\left(\frac{w}{(1-u)}\right)^{k} K_{10}\left(a_{1}+k, a_{1}+k, a_{1}+k, a_{1}+k, a_{2}, a_{2}, a_{3},\right. \\
& \left.c_{4}-a_{4} ; c_{1}, c_{2}, c_{3}, c_{4} ; \lambda_{1} x, \lambda_{1} y, \lambda_{1} z, \lambda_{2} u\right),
\end{aligned}
$$

where $\lambda_{1}=\frac{1}{1-u}, \lambda_{2}=\frac{1}{u-1}$.

$$
\begin{aligned}
\sum_{k=0}^{\infty} \frac{w^{k}}{k !} K_{10}\left(a_{1}+k, a_{1}+k, a_{1}+k, a_{1}+k, a_{2}, a_{2}, a_{3}, c_{4} ; c_{1}, c_{2}, c_{3}, c_{4} ; x, y, z, u\right) \\
=(1-z-u)^{-a_{1}} \sum_{k=0}^{\infty} \frac{1}{k !}\left(\frac{w}{(1-z-u)}\right)^{k} F_{E}\left(a_{1}+k, a_{1}+k, a_{1}+k,\right. \\
\left.c_{3}-a_{3}, a_{2}, a_{2} ; c_{3}, c_{1}, c_{2} ; \lambda_{2} z, \lambda_{1} x, \lambda_{1} y\right),
\end{aligned}
$$

where $\lambda_{1}=\frac{1}{1-z-u}, \lambda_{2}=\frac{1}{z+u-1}$.

$$
\begin{aligned}
\sum_{k=0}^{\infty} \frac{w^{k}}{k !} K_{10}\left(a_{1}+k, a_{1}+k, a_{1}+k, a_{1}+k, a_{2}, a_{2}, a_{3}, c_{4} ; c_{1}, c_{2}, c_{3}, c_{4} ; x, y, z, u\right) \\
=(1-u)^{-a_{1}} \sum_{k=0}^{\infty} \frac{1}{k !}\left(\frac{w}{(1-u)}\right)^{k} F_{E}\left(a_{1}+k, a_{1}+k, a_{1}+k, a_{3}, a_{2}, a_{2}\right. \\
\left.c_{3}, c_{1}, c_{2} ; \lambda z, \lambda x, \lambda y\right)
\end{aligned}
$$

where $\lambda=\frac{1}{1-u}$.

$$
\begin{gathered}
\sum_{k=0}^{\infty} \frac{w^{k}}{k !} K_{10}\left(a_{1}+k, a_{1}+k, a_{1}+k, a_{1}+k, c_{1}, c_{1}, a_{3}, a_{4} ; c_{1}, c_{1}, c_{3}, c_{4} ; x, y, z, u\right) \\
=(1-x-y-z-u)^{-a_{1}} \sum_{k=0}^{\infty} \frac{1}{k !}\left(\frac{w}{(1-x-y-z-u)}\right)^{k}
\end{gathered}
$$




$$
\begin{aligned}
& \times X_{8}\left(a_{1}+k, c_{3}-a_{3}, c_{4}-a_{4} ; c_{1}, c_{3}, c_{4} ;\right. \\
& \left.\quad \frac{x y}{(1-x-y-z-u)^{2}}, \frac{z}{(x+y+z+u-1)}, \frac{u}{(x+y+z+u-1)}\right),
\end{aligned}
$$

$$
\begin{aligned}
\sum_{k=0}^{\infty} \frac{w^{k}}{k !} K_{10}\left(a_{1}+k, a_{1}+k, a_{1}+k, a_{1}+k, c_{1}, c_{1}, a_{3}, a_{4} ; c_{1}, c_{1}, c_{3}, c_{4} ; x, y, z, u\right) \\
=(1-x-y)^{-a_{1}} \sum_{k=0}^{\infty} \frac{1}{k !}\left(\frac{w}{(1-x-y)}\right)^{k} X_{8}\left(a_{1}+k, a_{3}, a_{4} ; c_{1}, c_{3}, c_{4}\right. \\
\left.\quad \frac{x y}{(1-x-y)^{2}}, \frac{z}{(1-x-y)}, \frac{u}{(1-x-y)}\right)
\end{aligned}
$$

$$
\begin{aligned}
\sum_{k=0}^{\infty} \frac{w^{k}}{k !} K_{10}\left(a_{1}+k, a_{1}+k, a_{1}+k, a_{1}+k, c_{1}, c_{1}, c_{3}, a_{4} ; c_{1}, c_{1}, c_{3}, c_{4} ; x, y, z, u\right) \\
=(1-x-y-z-u)^{-a_{1}} \sum_{k=0}^{\infty} \frac{1}{k !}\left(\frac{w}{(1-x-y-z-u)}\right)^{k} H_{4}\left(a_{1}+k, c_{4}-a_{4}\right. \\
\left.c_{1}, c_{4} ; \frac{x y}{(1-x-y-z-u)^{2}}, \frac{u}{(x+y+z+u-1)}\right)
\end{aligned}
$$$$
\sum_{k=0}^{\infty} \frac{w^{k}}{k !} K_{10}\left(a_{1}+k, a_{1}+k, a_{1}+k, a_{1}+k, c_{1}, c_{1}, c_{3}, a_{4} ; c_{1}, c_{1}, c_{3}, c_{4} ; x, y, z, u\right)
$$$$
=(1-x-y-z)^{-a_{1}} \sum_{k=0}^{\infty} \frac{1}{k !}\left(\frac{w}{(1-x-y-z)}\right)^{k} H_{4}\left(a_{1}+k, a_{4} ; c_{1}, c_{4} ;\right.
$$$$
\left.\frac{x y}{(1-x-y-z)^{2}}, \frac{u}{(1-x-y-z)}\right)
$$

$$
\begin{aligned}
\sum_{k=0}^{\infty} \frac{w^{k}}{k !} K_{10}\left(a_{1}+k, a_{1}+k, a_{1}+k, a_{1}+k, a_{2}, a_{2}, c_{3}, c_{4} ; c_{1}, c_{2}, c_{3}, c_{4} ; x, y, z, u\right) \\
=(1-z-u)^{-a_{1}} \sum_{k=0}^{\infty} \frac{1}{k !}\left(\frac{w}{(1-z-u)}\right)^{k} F_{4}\left(a_{1}+k, a_{2} ; c_{1}, c_{2}\right. \\
\left.\quad \frac{x}{(1-z-u)}, \frac{y}{(1-z-u)}\right)
\end{aligned}
$$

$$
\begin{aligned}
\sum_{k=0}^{\infty} \frac{w^{k}}{k !} & K_{10}\left(a_{1}+k, a_{1}+k, a_{1}+k, a_{1}+k, c_{1}, c_{1}, c_{3}, c_{4} ; c_{1}, c_{1}, c_{3}, c_{4} ; x, y, z, u\right) \\
= & (1-x-y-z-u)^{-a_{1}} \sum_{k=0}^{\infty} \frac{1}{k !}\left(\frac{w}{(1-x-y-z-u)}\right)^{k} \\
& \times{ }_{2} F_{1}\left(\frac{a_{1}+k}{2}, \frac{a_{1}+k+1}{2} ; c_{1} ; \frac{4 x y}{(1-x-y-z-u)^{2}}\right),
\end{aligned}
$$

where $X_{8}$ is the Exton function and $H_{4}$ is the Horn function defined, respectively, by 


$$
X_{8}\left(a_{1}, a_{2}, a_{3} ; c_{1}, c_{2}, c_{3} ; x, y, z\right)=\sum_{m, n, p=0}^{\infty} \frac{\left(a_{1}\right)_{2 m+n+p}\left(a_{2}\right)_{n}\left(a_{3}\right)_{p}}{\left(c_{1}\right)_{m}\left(c_{2}\right)_{n}\left(c_{3}\right)_{p}} \times \frac{x^{m}}{m !} \frac{y^{n}}{n !} \frac{z^{p}}{p !}
$$

and

$$
H_{4}(a, b ; c, d ; x, y)=\sum_{m, n=0}^{\infty} \frac{(a)_{2 m+n}(b)_{m}}{(c)_{m}(d)_{n}} \frac{x^{m}}{m !} \frac{y^{n}}{n !}
$$

\section{Proofs of the results}

To prove the above relations, we need the following formulae (cf. $[4,7,9,10])$ :

$$
\begin{gathered}
\Gamma(z)=s^{z} \int_{0}^{\infty} e^{-s t} t^{z-1} d t, \quad \operatorname{Re}(z)>0, \\
(a)_{n}=\frac{\Gamma(a+n)}{\Gamma(a)}, \quad a \neq 0,-,-2, \cdots, \\
{ }_{1} F_{1}(-;-; x)=e^{x}, \\
{ }_{1} F_{1}(a ; b ; x)=e^{x}{ }_{1} F_{1}(b-a ; b ;-x), \\
\Psi_{2}(c, c ; c ; x, y)=\exp (x+y)_{0} F_{1}(-; c ; x y) .
\end{gathered}
$$

Proof of (2.1)

Let us denote the left-hand side of (2.1) by $\omega$, using (1.3)

$$
\begin{gathered}
\omega=\sum_{k=0}^{\infty} \frac{w^{k}}{k ! \Gamma\left(a_{1}+k\right)} \int_{0}^{\infty} e^{-s} s^{a_{1}+k-1} \Psi_{2}\left(a_{2} ; c_{1}, c_{2} ; s x, s y\right)_{1} F_{1}\left(a_{3} ; c_{3} ; s z\right) \\
\times{ }_{1} F_{1}\left(a_{4}, c_{4}, s u\right) d s,
\end{gathered}
$$

by using (3.4), we get

$$
\begin{aligned}
\omega=\sum_{k=0}^{\infty} & \frac{w^{k}}{k ! \Gamma\left(a_{1}+k\right)} \int_{0}^{\infty} e^{-s(1-z-u)} s^{a_{1}+k-1} \\
& \times \Psi_{2}\left(a_{2} ; c_{1}, c_{2} ; s x, s y\right)_{1} F_{1}\left(c_{3}-a_{3} ; c_{2} ;-s z\right)_{1} F_{1}\left(c_{4}-a_{4}, c_{4},-s u\right) d s .
\end{aligned}
$$

The functions $\Psi_{2}$ and ${ }_{2} F_{1}$ in the integrand can be written in its series form and, then interchanging the order of the summation and integral sign, which is permissible here, we get

$$
\begin{gathered}
\omega=\sum_{k, m, n, p=0}^{\infty} \frac{\left(a_{2}\right)_{m+n}\left(c_{3}-a_{3}\right)_{p}\left(c_{4}-a_{4}\right)_{q} w^{k} x^{m} y^{n}(-z)^{p}(-u)^{q}}{\left(c_{1}\right)_{m}\left(c_{2}\right)_{n}\left(c_{3}\right)_{p}\left(c_{4}\right)_{q} k ! m ! n ! p ! q ! \Gamma\left(a_{1}+k\right)} \\
\times \int_{0}^{\infty} e^{-s(1-z-u)} s^{a_{1}+k+m+n+p+q-1} d s .
\end{gathered}
$$

Now, use of (3.1) and (3.2) in the above equation, simplified with a series of manipulations, completes the proof of relation (2.1). The proofs of all remaining relations run in the same way, considering the appropriate integral representation and Laplace transform during the proof. 


\section{Special cases}

On the other hand, if we set $k=0$, in (2.1), (2.4), (2.6), (2.7), (2.9) and (2.10), we get the following interesting transformations formulas after little simplification:

$$
\begin{aligned}
& K_{10}\left(a_{1}, a_{1}, a_{1}, a_{1}, a_{2}, a_{2}, a_{3}, a_{4} ; c_{1}, c_{2}, c_{3}, c_{4} ; x, y, z, u\right) \\
& =(1-z-u)^{-a_{1}} \times K_{10}\left(a_{1}, a_{1}, a_{1}, a_{1}, a_{2}, a_{2}, c_{3}-a_{3}, c_{4}-a_{4} ; c_{1}, c_{2}, c_{3}, c_{4} ;\right. \\
& \left.\frac{x}{(1-z-u)}, \frac{y}{(1-z-u)}, \frac{z}{(z+u-1)}, \frac{u}{(z-u+1)}\right) \text {, } \\
& K_{10}\left(a_{1}, a_{1}, a_{1}, a_{1}, a_{2}, a_{2}, a_{3}, c_{4} ; c_{1}, c_{2}, c_{3}, c_{4} ; x, y, z, u\right) \\
& =(1-u)^{-a_{1}} \times F_{E}\left(a_{1}, a_{1}, a_{1}, a_{3}, a_{2}, a_{2} ; c_{3}, c_{1}, c_{2} ; \frac{z}{(1-u)}, \frac{x}{(1-u)}, \frac{y}{(1-u)}\right), \\
& K_{10}\left(a_{1}, a_{1}, a_{1}, a_{1}, c_{1}, c_{1}, a_{3}, a_{4} ; c_{1}, c_{1}, c_{3}, c_{4} ; x, y, z, u\right) \\
& =(1-x-y)^{-a_{1}} \times X_{8}\left(a_{1}, a_{3}, a_{4} ; c_{1}, c_{3}, c_{4} ; \frac{x y}{(1-x-y)^{2}}, \frac{z}{(1-x-y)}, \frac{u}{(1-x-y)}\right), \\
& K_{10}\left(a_{1}, a_{1}, a_{1}, a_{1}, c_{1}, c_{1}, c_{3}, a_{4} ; c_{1}, c_{1}, c_{3}, c_{4} ; x, y, z, u\right) \\
& =(1-x-y-z-u)^{-a_{1}} H_{4}\left(a_{1}, c_{4}-a_{4} ; c_{1}, c_{4} ; \frac{x y}{(1-x-y-z-u)^{2}},\right. \\
& \left.\frac{u}{(x+y+z+u-1)}\right) \\
& K_{10}\left(a_{1}, a_{1}, a_{1}, a_{1}, a_{2}, a_{2}, c_{3}, c_{4} ; c_{1}, c_{2}, c_{3}, c_{4} ; x, y, z, u\right) \\
& =(1-z-u)^{-a_{1}} \times F_{4}\left(a_{1}, a_{2} ; c_{1}, c_{2} ; \frac{x}{(1-z-u)}, \frac{y}{(1-z-u)}\right), \\
& K_{10}\left(a_{1}, a_{1}, a_{1}, a_{1}, c_{1}, c_{1}, c_{3}, c_{4} ; c_{1}, c_{1}, c_{3}, c_{4} ; x, y, z, u\right) \\
& =(1-x-y-z-u)_{2}^{-a_{1}} F_{1}\left(\frac{a_{1}}{2}, \frac{a_{1}+1}{2} ; c_{1} ; \frac{4 x y}{(1-x-y-z-u)^{2}}\right) \text {. }
\end{aligned}
$$

By setting $x=y=0$ in (4.2), we obtain the known result [9, p. 306 (109)]. Put $x=0$ in (2.10), we have generating function for Lauricella function $F_{A}^{(3)}$

$$
(1-y-z-u)^{a_{1}} e^{\left(\frac{w}{1-y-z-u}\right)}=\sum_{k=0}^{\infty} \frac{w^{k}}{k !} F_{A}^{(3)}\left(a_{1}+k, c_{1}, c_{3}, c_{4} ; c_{1}, c_{3}, c_{4} ; y, z, u\right) .
$$

Setting $u=0$ in (4.7), we shall obtain a generating function involving Appell function $F_{2}$

$$
(1-y-z)^{a_{1}} e^{\left(\frac{w}{1-y-z}\right)}=\sum_{k=0}^{\infty} \frac{w^{k}}{k !} F_{2}\left(a_{1}+k, c_{1}, c_{3} ; c_{1}, c_{3} ; y, z\right),
$$


which, for $z=0$, we get

$$
(1-y)^{a_{1}} e^{\left(\frac{w}{1-y}\right)}=\sum_{k=0}^{\infty} \frac{w^{k}}{k !}{ }_{2} F_{1}\left(a_{1}+k, c_{1} ; c_{1} ; y\right) .
$$

Finally, substituting $u=0$ in (2.5) and (2.8), we get the generating relations

$$
\begin{aligned}
& \sum_{k=0}^{\infty} \frac{w^{k}}{k !} F_{E}\left(a_{1}+k, a_{1}+k, a_{1}+k, a_{3}, c_{1}, c_{1} ; c_{3}, c_{1}, c_{1} ; z, x, y\right) \\
&=(1-x-y-z)^{-a_{1}} \sum_{k=0}^{\infty} \frac{1}{k !}\left(\frac{w}{(1-x-y-z)}\right)^{k} H_{4}\left(a_{1}+k, c_{3}-a_{3} ; c_{1}, c_{3} ;\right. \\
&\left.\frac{x y}{(1-x-y-z)^{2}}, \frac{z}{(x+y+z-1)}\right)
\end{aligned}
$$

and

$$
\begin{aligned}
& \sum_{k=0}^{\infty} \frac{w^{k}}{k !} F_{E}\left(a_{1}+k, a_{1}+k, a_{1}+k, c_{3}, c_{1}, c_{1} ; c_{3}, c_{1}, c_{1} ; z, x, y\right) \\
&=(1-x-y-z)^{-a_{1}} \sum_{k=0}^{\infty} \frac{1}{k !}\left(\frac{w}{(1-x-y-z)}\right)_{2}^{k} F_{1}\left(\frac{a_{1}+k}{2}, \frac{a_{1}+k+1}{2} ; c_{1} ;\right. \\
&\left.\frac{4 x y}{(1-x-y-z)^{2}}\right)
\end{aligned}
$$

respectively.

\section{Acknowledgements}

We are thankful to the editor and referees for their careful reading and valuable suggestions to make the article friendly readable. The research work of Praveen Agarwal is supported by Science and Engineering Research Board under Teachers Associateship for Research Excellence (TAR/2018/000001).

\section{References}

[1] Agarwal, P. \& Koul, C. L. (2003). On generating functions. J. Rajasthan Acad. Phy. Sci., 2, 173-180.

[2] Agarwal, P., Chand, M. \& Purohit, S. D. (2014). A note on generating functions involving the generalized Gauss hypergeometric function. National Acad. Sci. Lett., 3, 457-459.

[3] Choi, J. \& Agarwal, P. (2014). Certain generating functions involving Appell series. Far East J. Math. Sci., 84, 25-32.

[4] Erdelyi , A., Magnus, W., Oberhettinger, F. \& Tricomi, F. G. (1953). Higher Transcendental Functions. Vol. I, McGraw-Hill Book Company, New York, Toronto and London. 
[5] Exton, H. (1976). Multiple Hypergeometric Functions and Applications. Halsted Press, New York, London, Sydney and Toronto.

[6] Lauricella, G. (1893). Sull funzioni ipergeometric a più variabili. Rend. Cric. Mat. Palermo, 7, 111-158.

[7] Rainville, E. D. (1960). Special Functions. Chelsea Pub. Com., New York.

[8] Srivastava, H. M., Agarwal, P. \& Jain, S. (2014). Generating functions for the generalized Gauss hypergeometric functions. Appl. Math. Comput., 247, 348-352.

[9] Srivastava, H. M. \& Karlsson, P. W. (1985). Multiple Gaussian Hypergeometric Series. Halsted Press, Bristone, London, New York and Toronto.

[10] Srivastava, H. M. \& Manocha, H. L. (1984). A Treatise on Generating Functions. Ellis Horwood Lt1., Chichester. 